\title{
ON SUMMABILITY FIELDS OF CONSERVATIVE OPERATORS
}

BY H. I. BROWN, ${ }^{1}$ J. P. CRAWFORD AND H. H. STRATTON ${ }^{2}$

Communicated by John W. Green, February 4, 1969

Let $B[c]$ denote the Banach algebra of all bounded linear operators on $c$, the set of convergent sequences. By a conservative operator we mean a member of $B[c]$. If $T \in B[c]$ and if there exists an infinite matrix $A=\left(a_{n k}\right)$ such that $T x=A x$ for each $x \in c$, then $T$ is called a conservative matrix. (By $T x=A x$ we mean $(T x)_{n}=(A x)_{n} \equiv \sum_{k} a_{n k} x_{k}$ for each $n \in I^{+}$, the set of positive integers.) Let $\Gamma$ denote the subalgebra of $B[c]$ of all conservative matrices. If $T \in \Gamma$, its summability field, denoted by $c_{T}$, is taken to be the set $\{x \in s: T x \in c\}$, where $s$ denotes the set of all sequences. This raises the following question: How can one define the summability field $c_{T}$ for an arbitrary $T$ in $B[c]$ ? In other words, which sequences should one distinguish as being the set that a conservative operator sums?

One viewpoint is to consider how $T$ acts on $c_{0}$, the maximal subspace of $c$ consisting of those sequences which converge to 0 . The restriction of $T$ to $c_{0}$ is always representable by a matrix. In other words, if $T^{\prime}$ denotes the restriction of $T$ to $c_{0}$, then there is an infinite matrix $B$ so that $T^{\prime} x=B x$ for each $x \in c_{0}$. Surely, the summability field of $T^{\prime}$ is the set $c_{B}=\{x \in s: B x \in c\}$. We now note that if $T$ is a conservative matrix, say $A$, then $A$ also represents the restriction of $T$ to $c_{0}$, i.e. $A=B$. Thus, it seems reasonable to require that $c_{T} \supseteq c_{B}$ for any conservative operator $T$, where $B$ is the matrix representing the restriction of $T$ to $c_{0}$. Since the unit sequence $e=(1,1,1, \cdots)$ need not belong to $c_{B}$, even though $T e$ always belongs to $c$, we cannot, in general, take $c_{T}=c_{B}$. However, since $e$ is the only basis element of $c$ that $B$ might not sum, we propose that $c_{T}$ be defined as

$$
c_{T}=c_{B} \oplus e,
$$

where $\oplus$ denotes the linear span of the sets $c_{B}$ and $e$. The purpose then of this announcement is to report how the properties of $c_{T}$ defined above for $T \in B[c]$ compare with the well-known properties of $c_{T}$ for $T \in \Gamma$.

\footnotetext{
1 Research supported in part by NSF Grant GP-8199.

${ }^{2}$ Research supported in part by NSF Grant GP-8502.
} 
Further evidence supporting the choice of the above definition of $c_{T}$ is found in the following remarks. An $F K$ space (i.e., locally convex Fréchet sequence space with continuous coordinates [5]) which contains $c$ is called conull if $e$ belongs to the weak closure of $c_{0}$; otherwise, it is called coregular. Those $T \in \Gamma$ for which $c_{T}$ is conull are characterized by belonging to the kernel of the only nonzero multiplicative linear functional on $\Gamma$, denoted by $\chi$. ( $\chi$ is defined in $\S 1$ below.) The problem of extending the concept of conullity from $\Gamma$ to all of $B[c]$ was dealt with in an earlier paper [1]. It was shown there that there is exactly one subalgebra of $B[c]$, denoted by $\Omega$, which properly includes $\Gamma$, and that $\chi$ has a unique extension, denoted by $\rho$, to a nonzero multiplicative linear functional on $\Omega$. The kernel of $\rho$, therefore, became the natural definition for conullity in $B[c]$. We note here that the kernel of $\rho$ is precisely the set of those conservative operators $T$ for which $c_{T}$, as defined above, is a conull $F K$ space.

1. Further definitions and terminology. For each $k \in I^{+}$, let $e^{k}$ denote the sequence having one in the $k$ th coordinate and zeros elsewhere. If $x \in c, \lim x$ means $\lim _{i} x_{i}$. On $B[c]$ we have the functions

$$
\chi(T)=\lim T e-\sum_{k} \lim T e^{k}
$$

and

$$
\chi_{i}(T)=(T e)_{i}-\sum_{k}\left(T e^{k}\right)_{i}
$$

for each $i \in I^{+}$. (The functions $\chi, \chi_{i}$ are defined in [4]. See also [1].) It was pointed out in [4] that $\Gamma$ is precisely the set of those conservative operators $T$ for which $\chi_{i}(T)=0$ for every $i \in I^{+}$. The set of those conservative operators for which $\lim _{i} \chi_{i}(T)$ exists is denoted by $\Omega$. The structure of the subalgebras $\Gamma$ and $\Omega$ was studied in [1]. As was observed there, we may write each $T \in B[c]$ as follows:

$$
T x=(\lim x) v+B x \quad(\text { for } x \in c)
$$

where $v=\left\{\chi_{i}(T)\right\}$ and $B$ is the matrix representing the restriction of $T$ to $c_{0}$. This relationship between $T, v$ and $B$ will be denoted by $T \sim(v, B)$. We remark here that if $T \in \Omega$, then $B \in \Gamma$, while if $T \notin \Omega$, then $v \in m \backslash c$ (where $m$ denotes the set of bounded sequences), $B: c_{0} \rightarrow C$, and $e \notin c_{B}$.

2. The summability field $c_{T}$ and its dual space $c_{T}^{\prime}$. Let $T \in B[c]$, say $T \sim(v, B)$. Since $c_{B}$ is always an $F K$ space [5, p. 228], so is $c_{T}$. Define a functional $\alpha$ on $c_{T}$ as follows: If $T \in \Omega$ take $\alpha \equiv 0$, while if 
$T \notin \Omega$, let $\alpha(e)=1$ and $\alpha(x)=0$ for each $x \in c_{B}$. Then $\alpha$ is a continuous linear functional on $c_{T}$, i.e. $\alpha \in c_{T}^{\prime}$. Now let

$$
S=\{x \in s: \alpha(x) \cdot v+B x \in c\} .
$$

Since $B: m \rightarrow m$ and $v \in m$ we always have $m_{B} \supset S$, where

$$
m_{B}=\{x \in s: B x \in m\} .
$$

But $B$ is continuous as a map from $m_{B}$ into $m$ [5, Corollary 5, p. 204], $S=B^{-1}(c \oplus v)$, and $c \oplus v$ is a closed subspace of $m$; hence, $S$ is a closed subspace of $m_{B}$. Thus, $S$ is an $F K$ space [5, p. 203]. Now, by defining $d_{T}$, the domain of $T$, to be the set $\{x \in s: B x \in s\}$, a straightforward application of $\left[5\right.$, Theorems 5 and 6, p. 230] reveals that each $f \in S^{\prime}$, the dual space of $S$, has the representation

$$
\begin{aligned}
f(x)= & \delta \cdot \alpha(x)+d_{0} \cdot \lim _{n}\left(v_{n} \cdot \alpha(x)+\sum_{k} b_{n k} x_{k}\right) \\
& +\sum_{n} d_{n}\left(v_{n} \cdot \alpha(x)+\sum_{k} b_{n k} x_{k}\right)+\sum_{k} \beta_{k} x_{k},
\end{aligned}
$$

where $\beta=\left\{\beta_{k}\right\} \in s, \sum\left|d_{n}\right|<\infty$, and $d_{0}$ and $\delta$ are scalars, and $\alpha$ is the functional defined above. Moreover, since the kernel of $\alpha$ is precisely $c_{B}$ we see that $S=c_{T}$. Finally, since $c_{B} \supset c_{0}$ we see that $c_{T}$ is coregular whenever $T \notin \Omega$. We summarize these remarks in the following theorem.

THEOREM 1. For any conservative operator $T, c_{T}$ is an $F K$ space and the most general continuous linear functional on $c_{T}$ is given by equation (1). Moreover, if $T \notin \Omega$, then $c_{T}$ is coregular.

3. Some properties of $c_{T}$. A well-known result for matrix summability fields is that they cannot be properly contained between $c$ and $m$ [5, Problem 31, p. 231]. This property is not retained by $c_{T}$, as the following example illustrates.

Let $B$ be defined by the set of equations

$$
\begin{array}{ll}
b_{n n}=(-1)^{n+1}, & n=1,2, \cdots, \\
b_{2 n, 2 n-1}=1, & n=1,2, \cdots, \\
b_{n k}=0, & \text { otherwise. }
\end{array}
$$

Then $c_{B}=c_{0} \oplus y$, where $y=\{1,0,1,0, \cdots\}$ and hence if we set $v=\{0,1,0,1, \cdots\}$, then $T \sim(v, B)$ defines a conservative operator such that $c_{T}=c \oplus y$. 
The following theorem sheds some light on the structure of $c_{T}$ when it is a subset of $m$.

THEOREM 2. If $c_{T} \subset m$, either $c_{T}=c$ or $c$ is a maximal closed subspace of $c_{T}$.

Proof. If $T \in \Omega$, then $c_{T}=c_{B}$ and $B \in \Gamma$, and so $c_{T}=c$ whenever $c_{T} \subset m$. Suppose $T \notin \Omega$ and $c_{T} \subset m$. Then the topology of $c_{T}$ is the same as that of $m$ [5, Corollary 1, p. 203] and so $c$ is closed in $c_{T}$ since it is closed in $m$ and they have the same topology.

Let $f \in c_{T}^{\prime}$ with $f\left(e^{k}\right)=0$ for each $k \in I^{+}$. Then the representation (1) yields

$$
\beta_{k}=-d_{0} b_{k}-\sum_{n} d_{n} b_{n k},
$$

where $b_{k}=\lim _{n} b_{n k}$. Substituting this back into (1) and recalling that $c_{T} \subset m$ and $\sum\left|d_{n}\right|<\infty$ we obtain

$$
\begin{aligned}
f(x)= & \delta \cdot \alpha(x)+d_{0} \cdot \lim _{n}\left(v_{n} \cdot \alpha(x)+\sum_{k} b_{n k} x_{k}\right) \\
& +\sum_{n} d_{n} v_{n} \alpha(x)-\sum_{k} d_{0} b_{k} x_{k} .
\end{aligned}
$$

If we now also assume that $f(e)=0$, then, by letting $x=e$ in (2) and using the fact that $\alpha(e)=1$, we see that

$$
0=\delta+d_{0} \cdot \lim _{n}\left(v_{n}+\sum_{k} b_{n k}\right)+\sum_{n} d_{n} v_{n}-\sum_{k} d_{0} b_{k} .
$$

Since

$$
\chi(T)=\lim _{n}\left(v_{n}+\sum_{k} b_{n k}\right)-\sum_{k} b_{k}
$$

and since we may add a convergent sequence to $\left\{v_{n}\right\}$ without changing $c_{T}$, we see that we may assume $\chi(T)=0$. (Indeed, set $v_{n}^{\prime}=v_{n}-\chi(T)$ for each $n \in I^{+}$and let $T^{\prime} \sim\left(v^{\prime}, B\right)$ to obtain $c_{T^{\prime}}=c_{T}$ and $\chi\left(T^{\prime}\right)=0$.) It follows that $\delta+\sum_{n} d_{n} v_{n}=0$. Letting

$$
\Lambda(x)=\lim _{n}\left(v_{n} \alpha(x)+\sum_{k} b_{n k} x_{k}\right)-\sum_{k} b_{k} x_{k}
$$

we see that every functional which vanishes on $c$ has the form $f(x)=d_{0} \cdot \Lambda(x)$, and so the proof is complete.

Another well-known result in summability is that a conservative matrix $A$ is compact (i.e., $\sum_{k}\left|a_{n k}\right|$ converges uniformly with respect 
to $n$ ) if and only if it is coercive (i.e., $m \subset c_{A}$ ). Theorem 3 below will show that this result extends to conservative operators.

LEMma. If $T \notin \Omega$, then $m \Phi c_{T}$.

Proof. Suppose that $m \subset c_{T}$. Then $B: c_{0} \rightarrow c$ and $c_{B} \oplus e \supset m$; hence, the proof of Schur's Theorem [3, p. 17] shows that $\sum_{k}\left|b_{n k}\right|$ converges uniformly with respect to $n$, that is, $B$ is a compact operator on $m$.

Let $z^{1}, z^{2}, \cdots$ be a bounded set in $c$. Then

$$
v \cdot \alpha\left(z^{1}\right)+B z^{1}, v \cdot \alpha\left(z^{2}\right)+B z^{2}, \cdots
$$

is a subset of $c$. Let

$$
v \cdot \alpha\left(y^{1}\right)+B y^{1}, v \cdot \alpha\left(y^{2}\right)+B y^{2}, \cdots
$$

be any subsequence. Since $B$ is a compact operator on $m, B y^{1}$, $B y^{2}, \cdots$ has a convergent subsequence in $m$, say $B x^{1}, B x^{2}, \ldots$. Since $m \subset c_{T}, v \cdot \alpha(x)$ is also a compact operator on $m$, so $v \cdot \alpha\left(x^{1}\right)$, $v \cdot \alpha\left(x^{2}\right), \ldots$ also has a convergent subsequence in $m$, say $v \cdot \alpha\left(u^{1}\right)$, $v \cdot \alpha\left(u^{2}\right), \cdots$ Thus,

$$
v \cdot \alpha\left(u^{1}\right)+B u^{1}, v \cdot \alpha\left(u^{2}\right)+B u^{2}, \cdots
$$

is a subset of $c$ and converges, that is, $T$ is a compact operator. Since $c$ has a Schauder basis, $T$ is the uniform limit of operators with finite dimensional range. But each such operator belongs to $\Omega$. (For example, if $T$ has one dimensional range, say $T x=f(x) \cdot u$, where $u \in c$ and $f \in c^{\prime}$, then

$$
\chi_{i}(T)=\left(f(e)-\sum f\left(e^{k}\right)\right) \cdot u_{i}
$$

converges. The general case follows from this one.) It follows from the fact that $\Omega$ is closed in $B[c]$ that $T \in \Omega$. This proves the lemma.

TheOREM 3. $m \subset c_{T}$ if and only if $T$ is compact.

Proof. Suppose $T$ is compact. Then, as was pointed out in the proof of the lemma, $T \in \Omega$. It follows that $B$ is a compact conservative matrix and hence coercive. Thus, $m \subset c_{B}=c_{T}$.

Conversely, if $m \subset c_{T}$, then $T \in \Omega$. Hence, $c_{T}=c_{B}$ and $B$ is a compact conservative matrix. Since $v \in c, v \cdot \alpha(x)$ is also a compact operator on $c$. Thus, $T$ is compact.

For each $T \in B[c]$, say with $T \sim(v, B)$, we have already observed that there is associated a continuous linear functional $\alpha$ on $c_{T}$ so that

$$
c_{\boldsymbol{T}}=\{x \in s: v \cdot \alpha(x)+B x \in c\} .
$$


Thus, we may define

$$
\lim _{T} x=\lim _{n}\left(v_{n} \cdot \alpha(x)+\sum_{k} b_{n k} x_{k}\right)
$$

for $x \in c_{T}$. It is clear that $\lim _{T} \in c_{T}^{\prime}$. We say that $T$ satisfies the consistency property if the conditions $S \in B[c], c_{S} \supset c_{T}$ and $\lim _{T} x=\lim _{S} x$ on $c$ always imply that $\lim _{T} x=\lim _{S} x$ on $c_{T}$. We remark here that the procedure developed by Mazur [2] for use with conservative matrices can readily be adapted to conservative operators, and yields the following consistency-type result.

TheOREM 4. A conservative operator $T$ satisfies the consistency property if and only if $c$ is dense in $c_{T}$.

\section{REFERENCES}

1. H. I. Brown, D. R. Kerr and H. H. Stratton, The structure of $B[c]$ and extensions of the concept of connull matrix, Proc. Amer. Math. Soc. 22 (1969), 7-14.

2. S. Mazur, Eine Anwendung der Theorie der Operationen bei der Untersuchung der Toeplitzchen Limitierungsverfahren, Studia Math. 2 (1930), 40-50. 1966.

3. G. M. Petersen, Regular matrix transformations, McGraw-Hill, New York,

4. A. Wilansky, Topological divisors of zero and Tauberian theorems, Trans. Amer. Math. Soc. 113 (1964), 240-251.

5. —-, Functional analysis, Blaisdell, New York, 1964.

State University of New York, Albany, New York 12203 and

lafayette College, Easton, Pennsylvania 18042 